\title{
Quartic roots in ring of Gaussians
}

\author{
Thinh D. NGuYeN* \\ Moscow State University \\ kosmofarmer@gmail.com
}

July 24, 2018

\begin{abstract}
Taking square roots of -1 modulo a composite [3], taking quartic roots of 2 modulo a prime [4] are both shown to be hard tasks. Continuing this line of research with our friends Prasolov and the late I. F. Sharygin, we move on showing that computing quartic residue character in ring of Gaussian integrers can be solved if one can extend the known efficient factoring algorithms from integers to Gaussian integers.
\end{abstract}

\section{BACKGROUND}

In his second monograph on biquadratic reciprocity, Gauss displays some examples and makes conjectures that imply various theorems for biquadratic character of small primes. He makes some general remarks, and admits there is no obvious general rule at work. He goes on to say

The theorems on biquadratic residues gleam with the greatest simplicity and genuine beauty only when the field of arithmetic is extended to imaginary numbers, so that without restriction, the numbers of the form a + bi constitute the object of study... we call such numbers integral complex numbers.

These numbers are now called the ring of Gaussian integers, denoted by $\mathbb{Z}[i]$. Note that $i$ is a fourth root of 1 . In a foot note he adds

The theory of cubic residues must be based in a similar way on a consideration of numbers of the form $a+b h$ where $h$ is an imaginary root of the equation $h^{3}=1 \ldots$ and similarly the theory of residues of higher powers leads to the introduction of other imaginary quantities.

\footnotetext{
*Perebor
} 
The numbers built up from a cube root of unity are now called the ring of Eisenstein integers. The "other imaginary quantities" needed for the "theory of residues of higher powers" are the rings of the integers of the cyclotomic number fields; the Gaussian and Eisenstein integers are the simplest examples of these.

\section{FACTS AND TERMINOLOGY}

Gauss develops the arithmetics theory of the "integral complex numbers" and shows that it is quite similar to the arithmetic of ordinary integers. This is where the terms unit, associate, norm, and primary were introduced into mathematics. The units are the numbers that divide 1 . They are $1, i, 1$, and $i$. They are similar to 1 and 1 in the ordinary integers, in that they divide every number. The units are the powers of $i$. Given a number $\lambda=a+b i$, its conjugate is $a-b i$ and its associates are the four numbers

$$
\begin{aligned}
\lambda & =+a+b i \\
i \lambda & =-b+a i \\
-\lambda & =-a-b i \\
-i \lambda & =+b-a i
\end{aligned}
$$

The norm of $\lambda$, written $N \lambda$, is the number $a^{2}+b^{2}$. If $\lambda$ and $\mu$ are two Gaussian integers, $N \lambda \mu=N \lambda N \mu$; in other words, the norm is multiplicative. The norm of zero is zero, the norm of any other number is a positive integer. $\epsilon$ is a unit iff. $N \epsilon=1$. The square root of the norm of $\lambda$, a nonnegative real number which may not be a Gaussian integer, is the absolute value of $\lambda$. Gauss proves that $\mathbb{Z}[i]$ is a unique factorization domain and shows that the primes fall into three classes:

2 is a special case: $2=i^{3}(1+i)^{2}$. It is the only prime in $\mathbb{Z}$ divisible by the square of a prime in $\mathbb{Z}[i]$. In algebraic number theory, 2 is said to ramify in $\mathbb{Z}[i]$.

Positive primes in $\mathbb{Z} \equiv 3(\bmod 4)$ are also primes in $\mathbb{Z}[i]$. In algebraic number theory, these primes are said to remain inert in $\mathbb{Z}[i]$.

Positive primes in $\mathbb{Z} \equiv 1(\bmod 4)$ are the product of two conjugate primes in $\mathbb{Z}[i]$. In algebraic number theory, these primes are said to split in $\mathbb{Z}[i]$.

The associates and conjugates of a prime are also primes. Note that the norm of an inert prime $q$ is $N q=q^{2} \equiv 1 \bmod 4$. Gauss calls a number in $\mathbb{Z}[i]$ odd if its norm is an odd integer. Thus all primes except $1+i$ and its associates are odd. The product of two odd numbers is odd and the conjugate and associates of an odd number are odd. In order to state the unique factorization theorem, it is necessary to have a way of distinguishing one of the associates of a number. Gauss 
defines an odd number to be primary if it is $\equiv 1 \bmod (1+i)^{3}$. It is straightforward to show that every odd number has exactly one primary associate. An odd number $\lambda=a+b i$ is primary if $a+b \equiv a-b \equiv 1 \bmod 4$. The product of two primary numbers is primary and the conjugate of a primary number is also primary.

The unique factorization theorem for $\mathbb{Z}[i]$ is: if $\lambda \neq 0$, then $\lambda=i^{\mu}(1+i)^{v} \pi_{1}^{\alpha_{1}} \pi_{2}{ }^{\alpha_{2}} \pi_{3}{ }^{\alpha_{3}} \ldots$ where $0 \leq \mu \leq 3, v \geq 0$, the $\pi_{i}{ }^{\prime} \mathrm{s} \geq 1$, and this representation is unique up to the order of the factors.

The notion of congruence and greatest common divisor are defined the same way in $\mathbb{Z}[i]$ as they are the ordinary integers $\mathbb{Z}$. Because the units divide all numbers, a congruence $(\bmod \lambda)$ is also true modulo any associate of $\lambda$, and any associate of a GCD is also a GCD.

\section{i. Quartic residue character}

Gauss proves the analogue of Fermat's little theorem: if $\alpha$ is not divisible by an odd prime $\pi$, then $\alpha^{N \pi-1} \equiv \bmod \pi$

Since $N \pi \equiv 1 \bmod 4, \alpha^{\frac{N \pi-1}{4}}$ makes sense, and $\alpha^{\frac{N \pi-1}{4}} \equiv i^{k} \bmod \pi$ for a unique unit $i^{k}$. This unit is called the quartic or biquadratic residue character of $\alpha \bmod$ $\pi$ and is denoted by

$\left[\frac{\alpha}{\pi}\right]=i^{k} \equiv \alpha^{\frac{N \pi-1}{4}} \bmod \pi$

It has formal properties similar to those of Legendre symbol.

The congruence $x^{4} \equiv \alpha \bmod \pi$ is solvable in $\mathbb{Z}[i]$ iff. $\frac{\alpha}{\pi}=1$. This base case of quartic residuosity testing can be done efficiently and requiring no factorization. $\left[\frac{\alpha \beta}{\pi}\right]=\left[\frac{\alpha}{\pi}\right]\left[\frac{\beta}{\pi}\right]$

if $\pi$ and $\theta$ are associates, $\left[\frac{\alpha}{\pi}\right]=\left[\frac{\alpha}{\theta}\right]$. Also, if $\alpha \equiv \beta \bmod \pi,\left[\frac{\alpha}{\pi}\right]=\left[\frac{\beta}{\pi}\right]$

The biquadratic character can be extended to odd composites in the "denominator" in the same way the Legendre symbol is generalized into Jacobi symbol. As in that case, if the "denominator" is composite, the symbol can equal one without the congruence being solvable: $\left[\frac{\alpha}{\lambda}\right]=\left[\frac{\alpha}{\pi_{1}}\right]^{\alpha_{1}}\left[\frac{\alpha}{\pi_{2}}\right]^{\alpha_{2}} \ldots$ where $\lambda=\pi_{1}^{\alpha_{1}} \pi_{2}{ }^{\alpha_{2}} \pi_{3}{ }^{\alpha_{3}} \ldots$

It is now an appropriate time to have some necessary definition. Gaussian-Quartic-Residue-Character $=\left\{\langle\alpha, \lambda>|\left[\frac{\alpha}{\lambda}\right]=1\right\}$ 
So, now the following claim is immediate.

\section{ii. Claim}

Claim: Gaussian-Quartic-Residue-Character $\leq_{p}$ each member of NPC

If you somehow modify a blueprint of a practical computer to factorize Gaussian integers, you would have enough capabilities to compute quartic residue characters therein.

\section{REFERENCES}

[1] Euler, Leonhard (1849), Tractatus de numeroroum doctrina capita sedecim quae supersunt, Comment. Arithmet. 2

[2] Gauss, Carl Friedrich (1832), Theoria residuorum biquadraticorum, Commentatio secunda, Goettingen: Comment. Soc. regiae sci, Goettingen 7

[3] Thinh D. Nguyen, Taking square roots of -1, 2018

[4] Thinh D. Nguyen, Taking quartic roots of 2, 2018

[5] Quartic reciprocity, Wikipedia, the free encyclopedia

[6] Michael R. Garey, David S. Johnson, Computers and Intractability: A Guide to the Theory of NP-Completeness

[7] Phan Dinh Dieu, Le Cong Thanh, Le Tuan Hoa, Average Polynomial Time Complexity of Some NP-Complete Problems. Theor. Comput. Sci. 46(3): 219-237 (1986) 